\title{
Los roles gerenciales de Mintzberg: una evidencia empírica en la universidad
}

The managerial roles of Mintzberg: An empirical evidence in the university 


\title{
Los roles gerenciales de Mintzberg: una evidencia empírica en la universidad ${ }^{1}$
}

\section{The managerial roles of Mintzberg: An empirical evidence in the university}

\author{
Yulia Marcela Pacheco Galán², Yeisi Mar Molina Baquero³, \\ José Gregorio Arévalo Ascanio ${ }^{4}$ \\ 2,3,4 Universidad Francisco de Paula Santander Ocaña, Colombia
}

Artículo recibido en diciembre de 2016; artículo aceptado en abril de 2017

Citación del artículo: Pacheco, Y., Molina, Y. \& Arévalo, J. (2017). Los roles gerenciales de Mintzberg: una evidencia empírica en la universidad. I+D Revista de Investigaciones 10(2), julio - diciembre de 2017, pp. 102 - 111

\begin{abstract}
Resumen
Este artículo tiene como objetivo presentar el resultado de la evaluación del cumplimiento de los roles gerenciales planteados por Henry Mintzberg (interpersonales, de información y decisión) que predominan en los jefes de programas académicos de una institución de educación superior. La metodología utilizada se basó en un estudio cuantitativo, realizado por medio de la aplicación de encuestas individuales que permitieron analizar y presentar los resultados, los cuales evidencian que los roles predominantes en la unidad de análisis son los interpersonales y de información.
\end{abstract}

Palabras clave: rol, director, toma de decisiones, plan de estudios, universidad.

\section{Abstract}

This article aims to present the fulfillment of the managerial roles raised by Henry Mintzberg (interpersonal, information and decision) that predominate in the heads of academic programs of one institution of higher education. The methodology used was based on a quantitative study through the application of individual surveys that allowed the analysis and presentation of the results, which showed that the predominant roles in the unit of analysis are interpersonal and information.

Key words: role, director, decision making, curriculum, university.

1. Artículo de investigación terminada con enfoque cuantitativo, desarrollado por el grupo de investigación Gidse (categoría B de Colciencias) de la Universidad Francisco de Paula Santander, sede Ocaña.

2. Administradora de Empresas de la Universidad Francisco de Paula Santander (Ocaña). Dirección: Vía Acolsure, Sede el Algodonal, Ocaña (Norte de Santander) Orcid 0000-0002-4993-7801. Correo institucional: ympachecog@ufpso.edu.co.

3. Administradora de Empresas de la Universidad Francisco de Paula Santander (Ocaña). Dirección: calle 13 n. . 15-77. Orcid: 0000-0003-4591-3869. Correo institucional:ymmolinab@ufpso.edu.co.

4. Administrador de Empresas de la Universidad Francisco de Paula Santander (Ocaña). Magíster en Administración de Empresas de la Universidad Santo Tomás (Bucaramanga). Docente- investigador del grupo Desarrollo Socio Empresarial Gidse. Dirección: Universidad Francisco de Paula Santander, Ocaña (Colombia), Vía Acolsure, Sede Algodonal, PBX: 5690088 ext.: 484. Orcid 0000-0001-5464-2293. Correo institucional: jgarevaloa@ ufpso.edu.co. 
El directivo está sobrecargado de obligaciones; aun así, no puede delegar fácilmente sus tareas. En consecuencia, se ve obligado a trabajar en exceso y a realizar demasiadas tareas superficialmente; por tanto, el primer paso para proporcionar alguna ayuda al directivo consiste en descubrir lo que realmente es su trabajo (Minztberg, 1991). Dicho de otra manera, sin importar el nivel jerárquico que ocupen los directivos en cualquier organización, seguramente al consultarles sobre sus tareas, probablemente las asociarían con las funciones administrativas de planear, organizar, dirigir y controlar, es decir, lo que se conoce como la aplicación del proceso administrativo.

Ahora bien, en el marco del proyecto de investigación "Desempeño gerencial de los directores de planes de estudio de pregrado de la Universidad Francisco de Paula Santander, Ocaña", uno de cuyos objetivos fue analizar el cumplimiento de los roles gerenciales que predominan entre estos directivos, se aplicó un cuestionario a cada uno de los directores de programa académico de pregrado de esa institución.

El presente artículo se estructura así: una primera parte en la que se presenta una aproximación conceptual de los roles gerenciales de Henry Mintzberg, los cuales se agrupan en relaciones personales, transferencia de información y toma de decisiones. En la segunda parte se muestra que la metodología empleada fue de carácter cuantitativo a través de una investigación de campo en la que se aplicó un cuestionario con la escala de medición tipo Likert. La tercera parte analiza los resultados de la información recolectada, en los que se constata que a nivel general los directores desempeñan en un alto nivel los roles interpersonales, de información y de decisión; y por último, se exponen las principales conclusiones de la investigación.

\section{Los roles gerenciales: una mirada a Mintzberg}

En palabras de Mintzberg, la práctica gerencial se concibe como un triángulo donde se encuentran el arte, el oficio y el uso de la ciencia. El arte aporta las ideas y la integración; el oficio traza las conexiones; y la ciencia proporciona el orden por medio del análisis sistemático del conocimiento (Mintzberg, 2009). A continuación, se ilustra dicho triángulo (Figura 1).

Ahora bien, la dirección es la práctica diaria de administrar, dirigir o liderar organizaciones. Mintzberg considera estos conceptos difíciles de separar, ya que ocurren en forma mancomunada, quizás a veces de manera invisible, pero son necesarios para hacer comunidad en una organización (Levín, 2002).

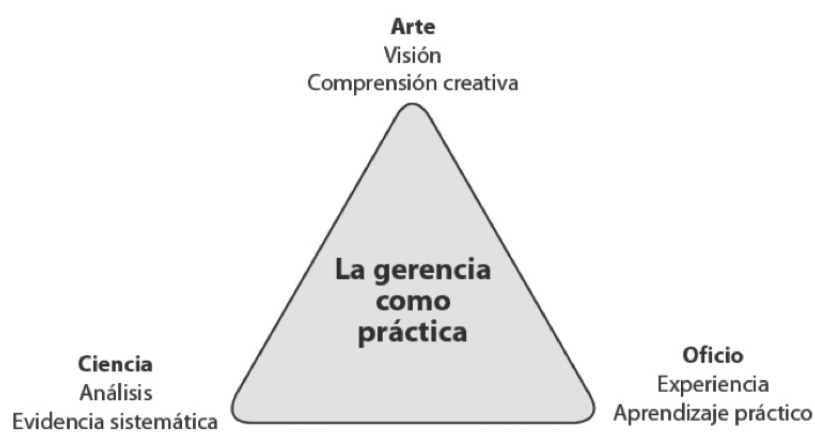

Figura 1.

La gerencia como arte, oficio y ciencia Fuente:Mintzberg, 2009

Por su parte Stoner, Freeman y Gilbert, señalan que el desempeño gerencial es la medida de la eficiencia y la eficacia de un gerente; grado en el que se alcanzan los objetivos acertados (2013, p. 47).

Paralelamente, Gudiño y Viloria (2010) afirman que el desempeño gerencial se mide por la eficiencia y eficacia de los directivos, la cuales se encuentran sustentadas en la capacidad de desarrollar un clima organizacional sinérgico, establecer y mantener una cultura organizacional con visión compartida, mantener un adecuado manejo de la información y tomar decisiones que garanticen la optimización organizacional.

Mientras tanto, Henry Mintzberg sostiene que los gerentes desempeñan diez roles muy relacionados, que se agrupan en interpersonales, de transferencia de información y de toma de decisiones. Los roles interpersonales son aquellos que tienen que ver con la gente y otros deberes de índole protocolaria y simbólica. Los roles informativos consisten en recibir, almacenar y difundir información y, por último, los roles de decisión giran en torno a la toma de decisiones. A continuación, se muestra una descripción de cada uno de los roles en la Tabla 1. 
Tabla 1

Roles gerenciales de Mintzberg

Rol

Interpersonal

Figura de autoridad

Líder

Enlace

Informativo

Supervisor

Difusor

Vocero

De decisión

Empresario

Manejador de perturbaciones

Distribuidor de recursos

Negociador
Descripción

Jefe simbólico; obligado a realizar deberes rutinarios de índole legal o social

Responsable de la motivación de los subordinados; responsable de reunir actividades que se refieren al personal, capacitarlo y deberes relacionados.

Crea y mantiene una red de contactos externos e informadores que le hacen favores y le dan información.

Busca y recibe amplia información interna y externa para comprender a fondo la organización y el ambiente.

Transmite la información recibida de fuera o de los subordinados a los miembros de la organización.

Transmite información a gente de fuera sobre los planes de la organización, políticas, acciones, resultados, etcétera.

Busca oportunidades en la organización y el entorno e inicia "proyectos de mejora" para producir cambios.

Es responsable de las acciones correctivas cuando la organización enfrenta perturbaciones graves e inesperadas.

Es responsable de la asignación de todos los recursos de la organización; toma o aprueba todas las decisiones importantes de la organización.

Es responsable de representar a la organización en las principales negociaciones.

\section{Fuente: Autores}

\section{Investigaciones sobre desempeño gerencial en el contexto educativo}

Con el objetivo de explorar los estudios sobre desempeño gerencial se realizó una revisión sistemática en Redalyc, Scielo y Science Direct, a partir de categorías como el desempeño gerencial, los roles gerenciales y los roles de Mintzberg, y limitándolos a los artículos publicados en revistas y tesis de universidades cuya temática fuera aplicable al contexto educativo. Finalmente, se encontraron siete documentos (empíricos) que se relacionaban de forma directa con las categorías planteadas.

Sánchez (2003) afirma, en su estudio sobre el estilo gerencial del personal directivo y docente de educación básica (escuelas básicas que funcionan en el municipio de Boconó del Estado de Trujillo en Venezuela), que la supervisión es de vital importancia en el proceso escolar, ya que a través de ella se pueden efectuar los correctivos a las posibles desviaciones e irregularidades que se presentan durante el proceso de supervisión, creando en el plantel un clima para desarrollar unas relaciones interpersonales favorables para el trabajo; además, evidenció en la gran mayoría de estos planteles un estilo gerencial democrático.

Bracho (2005) en su estudio titulado Desempeño gerencial: funciones y roles en la práctica, cuyo universo de estudio estuvo constituido por los profesionales egresados de la maestría en Gerencia Empresarial de la Universidad Centroccidental "Lisandro Alvarado" (UCLA), se encontró que en los egresados de la III cohorte de la maestría en Gerencia Empresarial del Decanato de Administración y Contaduría existe una marcada y positiva tendencia a asumir roles gerenciales de empresario y mediador de problemas inesperados, con los cuales se logran avances en las organizaciones al promover cambios necesarios y buscar soluciones a problemas importantes e inesperados. En contraposición, evidenció que existe una innegable debilidad derivada del bajo desempeño de roles de asignador de recursos y de negociador, siendo evidente que la no participación en procesos de asignación de recursos pone en situación de desventaja a los gerentes en actividades de negociación, limitando, en consecuencia, una labor efectiva. 
El trabajo de Borjas y Vera (2008) analiza las funciones gerenciales del director de las escuelas bolivarianas de la Parroquia Alonso de Ojeda (Estado Zulia, Venezuela); uno de los hallazgos más importantes de este estudio fue que se determinó que se debe evaluar el rol del gerente educativo por cuanto se observaron diferencias con respecto a la opinión de los directivos y de los docentes; además, se detectó que los directores, en su función gerencial, cumplen con la mayoría de las funciones solo en el control.

Matos y Caridad (2009) presentan un estudio orientado a determinar la relación entre las competencias gerenciales y el desempeño laboral de las autoridades en universidades nacionales experimentales en el Estado Zulia (Venezuela); en lo referido a los roles gerenciales concluyeron que los directores y docentes los llevan a cabo moderadamente, ejecutándose ciertas acciones que contribuyen al desarrollo de sus funciones, específicamente el rol de agente.

Frías, Sánchez y Ucrós (2009), mediante un estudio descriptivo no experimental, determinaron las habilidades gerenciales en los directivos de la Universidad de La Guajira; para ello seleccionaron una muestra no probabilística intencional formada por un total de veintisiete sujetos. Los resultados evidenciaron que los directivos universitarios poseen habilidades gerenciales asociadas con la autoestima, la comunicación, la participación, el desempeño y el liderazgo. Asimismo, los hallazgos mostraron que en los directivos existen destrezas técnicas, humanas y conceptuales en relación con las habilidades gerenciales, lo cual les permite ser asertivos en la toma de decisiones y mantener una efectiva comunicación con sus colaboradores.

Olaizola (2012) presentó un estudio que tuvo como propósito evaluar el clima organizacional y el desempeño gerencial del personal directivo hacia una gestión de calidad en la escuela básica Doctor Francisco Espejo, ubicada en Valencia, Estado Carabobo (Venezuela); entre las conclusiones más importantes se encuentra el hecho de que el gremio docente de esta institución percibe las acciones relacionadas con el desempeño gerencial de los directivos como insuficientes, ya que sus funciones se ven ampliamente influenciadas por los lineamientos emanados por la Secretaría de Educación y Deportes del Estado Carabobo, debilitando así la acción personal y autónoma de la que gozan como personal directivo.

Finalmente Turiño, Cañizares y Sarasa (2013), a través de un estudio descriptivo transversal con enfoque cualitativo realizado en doce jefes de departamentos docentes de la sede central de la Universidad de Ciencias
Médicas de Villa Clara (Cuba), identificaron que en los jefes objeto de estudio existen distintas necesidades de aprendizaje de carácter gerencial que pueden afectar la dirección del trabajo metodológico y científico y además se constituyen como una debilidad en las competencias gerenciales en dichos jefes.

\section{Método}

\section{Tipo de estudio}

Se realizó una investigación descriptiva, la cual según Hurtado (2000), tiene como objetivo central lograr la descripción o caracterización del evento de estudio dentro de un contexto particular, es así que el presente estudio se centró en el enfoque cuantitativo, en tanto que usó la recolección de datos, con base en la medición numérica y el análisis estadístico, para establecer patrones de comportamiento y probar teorías (Hernández, Fernández \& Baptista, 2006).

\section{Participantes}

La población seleccionada para esta investigación fueron los jefes de planes de estudio de pregrado de las diferentes facultades de la Universidad Francisco de Paula Santander, sede Ocaña, representada por una población finita de doce personas.

\section{Materiales e instrumentos}

Para recolectar la información se utilizó una encuesta diseñada por Alejandra Pernía en su investigación, titulada "Desempeño gerentes de nivel medio de los diferentes decanatos, en la Universidad Centro Occidental Lisandro Alvarado". Dicho instrumento contiene preguntas que ayudaron a determinar el desempeño de los directores de planes de estudio de pregrado. Cada cuestionario consta de dos partes; en la primera, se buscó determinar las funciones administrativas, y la segunda correspondió a la identificación de los roles gerenciales. Para identificar dichos roles se desarrolló una investigación de campo mediante la aplicación del cuestionario con la escala de medición tipo Likert.

\section{Procedimiento}

La información cuantitativa se procesó y se analizó mediante la elaboración de tablas y gráficos, tomando como base los datos obtenidos en la aplicación de las encuestas. Es preciso destacar que los datos obtenidos en las encuestas se presentan en forma global y no individual, con la finalidad de tener una visión integral sobre los aspectos estudiados. 


\section{Resultados}

El investigador Henry Mintzberg dice que la mejor manera de describir lo que hacen los gerentes es observar los roles que representan en el trabajo, los cuales se refieren a las categorías particulares del comportamiento administrativo. (Robbins \& Coulter, 2005)

Al analizar el cumplimiento de los roles gerenciales según Mintzberg, que predominan en los directores de planes de estudio de pregrado, es preciso indicar que para el análisis y procesamiento de la información se agruparon los ítems de la escala Likert estructurados en la encuesta de la siguiente manera: completamente de acuerdo y de acuerdo pasan a ser sí, completamente en desacuerdo y en desacuerdo se convierten en no e indeciso se cambia a no informa, ya que mediante este mecanismo se facilitó el análisis e interpretación de los papeles que desempeñan los directores de programa académico de pregrado; en la Tabla 2 se evidencia lo acontecido con el rol Interpersonal (es decir, roles asociados con la interacción del gerente con otros miembros de la organización: superiores, subordinados, iguales y personas externas a la organización).

A continuación, se muestran gráficamente los resultados obtenidos en relación con el rol Interpersonal (Figura 2).

Tabla 2

Desempeño del rol Interpersonal de los directores de planes de estudio de pregrado

\begin{tabular}{|c|c|c|c|c|c|}
\hline & Ítems & Sí & No & $\begin{array}{l}\text { No } \\
\text { informa }\end{array}$ & $\begin{array}{l}\text { No } \\
\text { responde }\end{array}$ \\
\hline \multirow{7}{*}{ 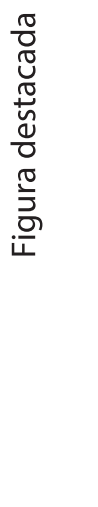 } & $\begin{array}{l}\text { 1. Representa socialmente a su unidad } \\
\text { (Director de programa) en su entorno. }\end{array}$ & $100 \%$ & 0 & 0 & 0 \\
\hline & $\begin{array}{l}\text { 2. La atención de visitantes a su unidad forma } \\
\text { parte de sus labores. }\end{array}$ & $83 \%$ & $17 \%$ & 0 & 0 \\
\hline & 3. Firma documentos legales de su unidad & $100 \%$ & & 0 & 0 \\
\hline & Total & $94 \%$ & $6 \%$ & 0 & 0 \\
\hline & $\begin{array}{l}\text { 4. El incentivo de su personal forma parte de } \\
\text { sus responsabilidades. }\end{array}$ & $83 \%$ & $17 \%$ & 0 & 0 \\
\hline & $\begin{array}{l}\text { 5. Participa en el proceso de contratación de } \\
\text { personal. }\end{array}$ & $25 \%$ & $67 \%$ & 0 & $8 \%$ \\
\hline & $\begin{array}{l}\text { 6. Participa en el proceso de evaluación del } \\
\text { personal. }\end{array}$ & $92 \%$ & $8 \%$ & 0 & 0 \\
\hline \multirow[t]{8}{*}{$\frac{\bar{d}}{\underline{\underline{o}}}$} & $\begin{array}{l}\text { 7. Participa en los procesos de capacitación y } \\
\text { entrenamiento del personal. }\end{array}$ & $83 \%$ & $17 \%$ & 0 & 0 \\
\hline & 8. Ejerce influencia entre su personal. & $83 \%$ & $17 \%$ & 0 & 0 \\
\hline & 9. Asume el liderazgo en su equipo de trabajo. & $92 \%$ & $8 \%$ & 0 & 0 \\
\hline & $\begin{array}{l}\text { 10.Permanentemente establece mecanismos } \\
\text { formales e informales para la solución de } \\
\text { conflictos. }\end{array}$ & $92 \%$ & $8 \%$ & 0 & 0 \\
\hline & Total & $79 \%$ & $20 \%$ & 0 & $1 \%$ \\
\hline & $\begin{array}{l}\text { 11. Actúa como enlace entre la organización } \\
\text { y el entorno. }\end{array}$ & $92 \%$ & $8 \%$ & 0 & 0 \\
\hline & 12. Recibe y responde correspondencia. & $100 \%$ & & 0 & 0 \\
\hline & $\begin{array}{l}\text { 13. Establece contactos externos para } \\
\text { mantenerse informado. }\end{array}$ & $76 \%$ & $8, \%$ & $8, \%$ & $8 \%$ \\
\hline \multirow[t]{2}{*}{ 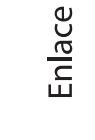 } & $\begin{array}{l}\text { 14. Establece y mantiene contactos externos } \\
\text { para agilizar sus operaciones. }\end{array}$ & $83 \%$ & $17 \%$ & 0 & 0 \\
\hline & Total & $88 \%$ & $8 \%$ & $2 \%$ & $2 \%$ \\
\hline
\end{tabular}

Fuente: Autores 


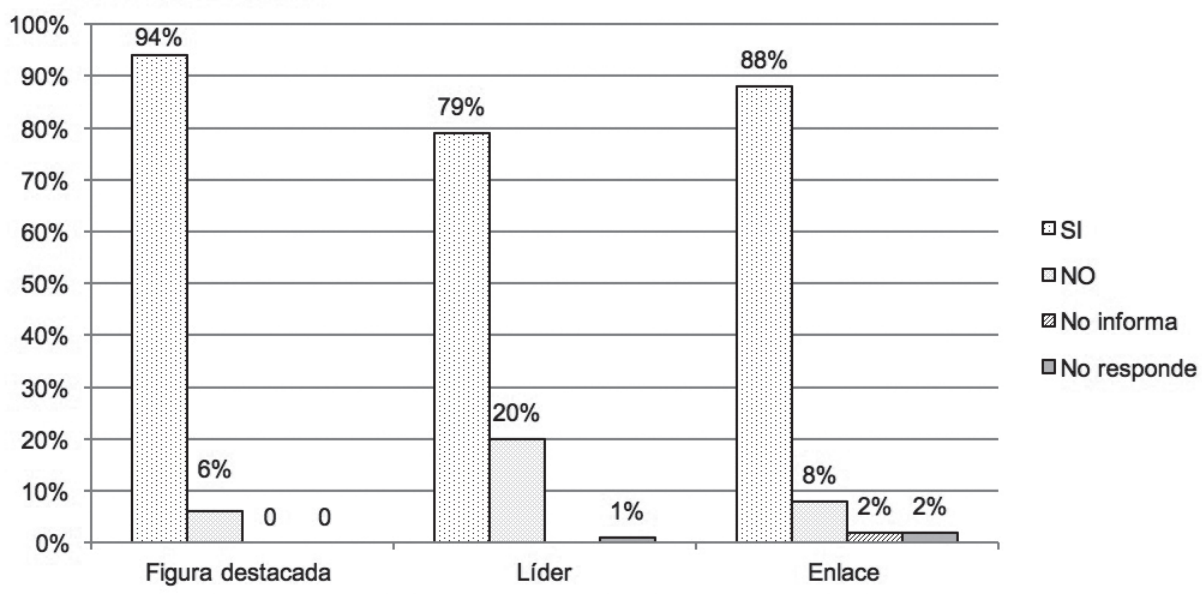

Figura 2.

Porcentaje de desempeño de los diferentes aspectos del rol Interpersonal de los directores de planes de estudio Fuente: Autores

En cuanto al rol Interpersonal se observa que los tres indicadores apuntan hacia un alto desempeño, ya que sobrepasan el $75 \%$. Sin embargo, se puede notar que el indicador más sobresaliente es el de "Figura destacada", debido a que la mayoría de los directores señalaron que entre sus funciones están la atención a visitas, la firma de documentos legales y la representación de la unidad en el entorno.

El indicador "Liderazgo" es el de menor ponderación, con $79 \%$, lo que se debe a que la mayoría de los directores afirma que entre sus funciones no está contratar el personal; no obstante, los directores participan activamente en el proceso de capacitación, entrenamiento del personal, solución de conflictos $y$ asumen el liderazgo en sus equipos de trabajo, variables que les permiten orientar a su personal hacia la consecución de los objetivos, garantizando el adecuado funcionamiento de la gestión administrativa académica. Finalmente, el indicador "Enlace", ponderado en un $88 \%$, muestra que los directores de planes de estudio establecen y mantienen contacto con el medio externo, una situación que es relevante en la toma de decisiones de sus unidades académico - administrativas, ya que los aspectos económicos, políticos, sociales, tecnológicos, ecológicos y culturales tienen un fuerte impacto sobre el desarrollo del programa académico (ver Tabla 3).

Tabla 3

Rol Información según los directores de planes de estudio de pregrado

\begin{tabular}{|c|c|c|c|c|}
\hline & Ítems & No & Sí & $\begin{array}{l}\text { No } \\
\text { informa }\end{array}$ \\
\hline 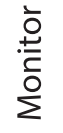 & $\begin{array}{l}\text { 15. Revisa permanentemente publicaciones e informes sobre la organización } \\
\text { y su entorno. }\end{array}$ & $100 \%$ & 0 & 0 \\
\hline $\begin{array}{l}\frac{\overline{0}}{0} \\
\frac{\pi}{0} \\
\frac{0}{3} \\
\frac{1}{0}\end{array}$ & $\begin{array}{l}\text { 16. Transmite información de sus área de influencia y del entorno a los } \\
\text { niveles superiores. }\end{array}$ & $100 \%$ & 0 & 0 \\
\hline $\begin{array}{l}\text { N } \\
\text { Ò } \\
\text { ț } \\
0\end{array}$ & $\begin{array}{l}\text { 17. La transmisión de información de la organización ha entes externos } \\
\text { forma parte de sus labores. }\end{array}$ & $75 \%$ & $25 \%$ & 0 \\
\hline
\end{tabular}

Fuente: Autores 
En cuanto al desempeño del rol informativo, este se analiza con base en los tres indicadores: monitor, divulgador y portavoz, los cuales arrojaron los siguientes resultados $100 \%, 100 \%$ y $75 \%$. Claramente se evidencia que los dos primeros indicadores son desempeñados en un alto nivel, ya que los directores ejercen acciones tales como la búsqueda permanente de información útil, tanto interna como externa, así como su divulgación entre sus colaboradores, facilitando el desempeño de sus funciones y lo cual se constituye en una parte importante dentro de sus labores. Mientras que del rol portavoz tiene una menor ponderación, acontecimiento que obedece a que la información trasmitida a entes importantes es compartida en gran medida con los decanos, quienes son considerados los jefes inmediatos de la unidad de análisis.

Partiendo de lo anterior, es clave que los administradores de los planes de estudio formen redes de contactos que les ofrezcan acceso a información importante, tal y como lo sugiere Mintzberg que los roles informativos son uno de los aspectos más importantes del trabajo de un administrador, son ellos quienes necesitan información para tomar decisiones oportunas e inteligentes. En la Tabla 4 se muestran los aspectos relacionados con el rol "Decisión".

Tabla 4

Rol "Decisión" según los directores de planes de estudio de pregrado

\begin{tabular}{|c|c|c|c|c|}
\hline & Ítems & No & Sí & $\begin{array}{l}\text { No } \\
\text { informa }\end{array}$ \\
\hline \multirow{6}{*}{ 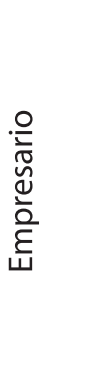 } & $\begin{array}{l}\text { 18. Detecta fortalezas en la organización } \\
\text { para promover cambios. }\end{array}$ & $92 \%$ & $8 \%$ & 0 \\
\hline & $\begin{array}{l}\text { 19. Detecta oportunidades en el entorno para promover } \\
\text { cambios. }\end{array}$ & $92 \%$ & $8 \%$ & 0 \\
\hline & 20. Emprende nuevos proyectos, programas o ideas. & $83 \%$ & $17 \%$ & 0 \\
\hline & 21. Inicia y supervisa el diseño de proyectos. & $92 \%$ & $8 \%$ & 0 \\
\hline & $\begin{array}{l}\text { 22. Considera que su trabajo requiere de innovación y } \\
\text { creatividad. }\end{array}$ & $92 \%$ & $8 \%$ & 0 \\
\hline & Total & $90 \%$ & $10 \%$ & 0 \\
\hline \multirow{4}{*}{ 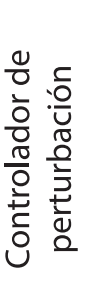 } & $\begin{array}{l}\text { 23. Soluciona contingencias ocurridas interna o } \\
\text { externamente. }\end{array}$ & $92 \%$ & $8 \%$ & 0 \\
\hline & $\begin{array}{l}\text { 24. Organiza espacios de discusión y revisión de problemas } \\
\text { inesperados. }\end{array}$ & $100 \%$ & 0 & 0 \\
\hline & $\begin{array}{l}25 . \text { Es responsable de tomar acciones correctivas cuando la } \\
\text { organización enfrenta situaciones inesperadas. }\end{array}$ & $75 \%$ & $8 \%$ & $17 \%$ \\
\hline & Total & $89 \%$ & $5 \%$ & $6 \%$ \\
\hline \multirow{3}{*}{ 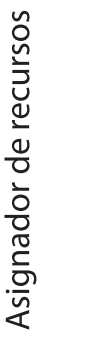 } & $\begin{array}{l}\text { 26. Participa en la programación de actividades de los } \\
\text { trabajadores. }\end{array}$ & $75 \%$ & $17 \%$ & $8 \%$ \\
\hline & $\begin{array}{l}\text { 27. Le corresponde asignar recursos (humanos, físicos y } \\
\text { financieros) para el logro de los objetivos previstos. }\end{array}$ & $50 \%$ & $42 \%$ & $8 \%$ \\
\hline & Total & $62 \%$ & $30 \%$ & $8 \%$ \\
\hline \multirow{3}{*}{$\begin{array}{l}\frac{1}{0} \\
\frac{0}{0} \\
\frac{\pi}{2} \\
\text { O্] } \\
\frac{d}{z}\end{array}$} & $\begin{array}{l}\text { 28. Representa externamente a la organización en procesos } \\
\text { de negociación. }\end{array}$ & $50 \%$ & $25 \%$ & $25 \%$ \\
\hline & $\begin{array}{l}\text { 29. Representa internamente a la organización de los } \\
\text { procesos de negociación. }\end{array}$ & $67 \%$ & $16 \%$ & $17 \%$ \\
\hline & Total & $58 \%$ & $21 \%$ & $21 \%$ \\
\hline
\end{tabular}

\section{Fuente: Autores}




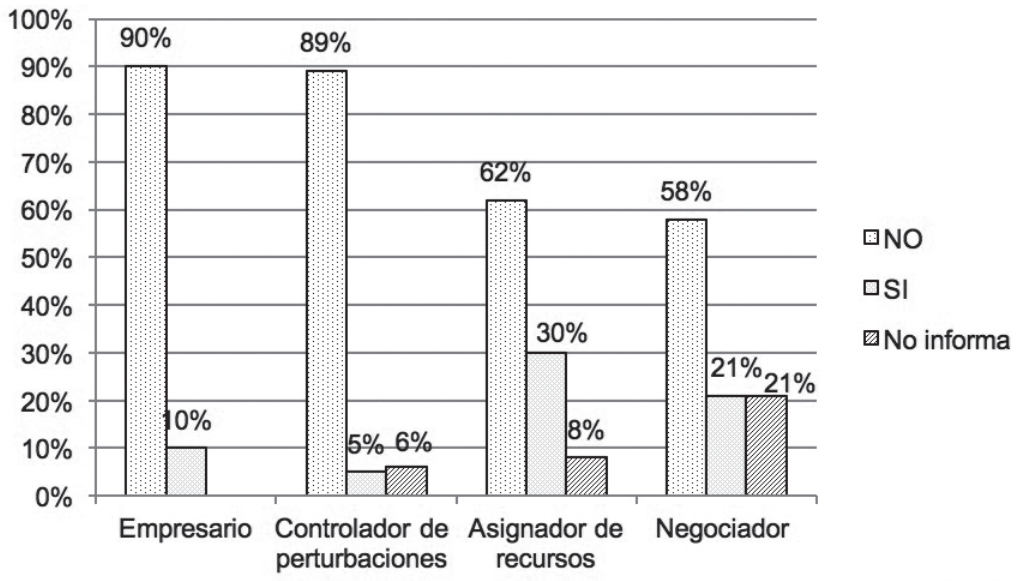

Figura 3.

Rol de decisión aplicado por los directores de planes de estudio.

Fuente: Autores

El rol "Decisión", como se pudo observar en el gráfico anterior, se distribuye en cuatro papeles: empresario, controlador de perturbación, asignador de recursos y negociador. Los dos primeros suman un porcentaje mayor al $85 \%$ y los dos últimos están por encima del $55 \%$, lo que indica que, en su mayoría, los directores de programa desempeñan este rol.

Se observa que el rol "Empresario" tiene una representación significativa $(90 \%)$ debido a que las actividades como innovar, crear, diseñary dar continuidad a nuevos proyectos son ejercidas por la mayor parte de los directores de pregrado; por su parte, en cuanto al rol "Control de perturbaciones", se evidencia que los directores realizan actividades tales como dar solución a contingencias y organizar espacios de discusión. Este rol muestra que los directores se ajustan con facilidad a los cambios y exigencias del entorno que pueden perturbar el desarrollo eficaz de las diferentes actividades.

En cuanto al rol "Asignador de recursos", se observa que la gran mayoría de los sujetos encuestados no asignan recursos financieros sino que los gestionan y distribuyen; en cuanto a los recursos humanos administrativos, tampoco no son designados por los directivos, ya que la universidad cuenta con un área de recursos humanos. Por último, el rol "Negociador" se desarrolla más internamente que externamente, esto se debe a que esta función es compartida en gran medida con los decanos, ya que en conjunto estos representan, desde las dos perspectivas (interna y externa), a los programas académicos en los procesos de negociación.
Finalmente, no cabe duda de que el rol de tomador de decisiones para los directores de planes de estudio es determinante en el accionar de cada uno de los programas, puesto que tomar decisiones es una de sus principales atribuciones en su papel de coordinador académico.

\section{Conclusiones}

Es indudable que los roles gerenciales necesitan ser tenidos en cuenta al momento de conducir un programa académico, así como sucede en una unidad estratégica de negocios de cualquier organización empresarial, pues tal conducción se materializa con la incorporación de diferentes roles que deben aportar para que la dirección administrativa se dé de manera acertada.

En cuanto a los roles gerenciales propuestos por Mintzberg se pudo constatar que, a nivel general, los directores de programa de pregrado desempeñan en un alto nivel los roles Interpersonal, Información y Decisión, de lo cual se puede inferir que los diferentes gerentes de los planes de estudio establecen vínculos con la gente, gestionan información y tienen habilidades para tomar decisiones. A nivel general, los roles gerenciales de Mintzberg predominantes en los directores consultados son Interpersonal e Información.

Los resultados muestran que los directores, como líderes de los procesos académicos, tienen una variedad de responsabilidades, es decir, su papel dentro de la universidad es multifacético, por lo tanto, sus tareas tienden a incluir una variedad de funciones como administradores (del tiempo, del ambiente de trabajo, de la visión curricular y de las relaciones públicas externas). 
En este sentido es importante que los directores de planes de estudios interioricen los roles gerenciales propuestos por Mintzberg, en el caso particular de la universidad objeto de estudio y teniendo en cuenta los resultados obtenidos frente al rol decisorio, se requiere de una gerencia más consciente de la importancia de la toma de decisiones como la variable determinante para garantizar el logro de los objetivos académicoadministrativos.

\section{Agradecimientos}

A la Universidad Francisco de Paula Santander, sede Ocaña, a través de la División de Investigación y Extensión DIE por su colaboración en la financiación del proyecto del cual se deriva el manuscrito. Se destaca el apoyo de la Facultad de Ciencias Administrativas y Económicas y específicamente, del plan de estudios de Administración de Empresas, programa académico del que hacen parte los autores involucrados en el proyecto.

\section{Referencias}

Borjas, F. \& Vera, L. (2008). Funciones gerenciales del director de las escuelas bolivarianas. Negotium, Revista Científica Electrónica Ciencias Gerenciales, 70-103.

Bracho, A. (2005). Desempeño gerencial: funciones y roles en la práctica. Compendium, 5-19.

Frías, E., Sánchez, D. \& Ucrós, M. (2009). Habilidades gerenciales en directivos. Caso de estudio: Universidad de la Guajira. Universidad Eafit, 94-102.

Gudiño, M. \& Viloria, N. (2010). Desempeño gerencial en las instituciones de la Avec del municipio Boconó. Telos. Revista de Estudios Interdisciplinarios en Ciencias Sociales, 43-62.

Hernández, R., Fernández, C. \& Baptista, P. (2006). Metodología de la investigación. México: Mc Graw Hill.

Hurtado, J. (2000). Metodología de la investigación holística. Caracas: Sypal.

Levín, S. (2002). Reseña Mintzberg y su tratamiento contemporáneo. Gestion y estrategia, 113-117.

Matos, G. \& Caridad, M. (2009). Competencias gerenciales y desempeño laboral de autoridades en universidades nacionales experimentales. Centro de Investigación de Ciencias Administrativas y Gerenciales Cicag, 6(1).

Minztberg, H. (1991). El trabajo directivo. Folklore y hechos, en Minztberg y la dirección. Madrid: Díaz de Santos S.A.
Olaizola, L. (2012). Clima organizacional y desempeño gerencial del personal directivo en la E. B. "Dr. Francisco Espejo". (Maestría de Gerencia Avanzada en Educación). Universidad de Carabobo, Carabobo.

Pernía, A. (2008). Desempeño gerencial de los gerentes de nivel medio de los diferentes decanatos, en la universidad centro occidental "Lisandro Alvarado". (Tesis de maestría). Barquisimeto.

Robbins, S. \& Coulter, M. (2005). Administración. México: Pearson.

Sánchez, E. (2003). Estilo gerencial del personal directivo y relaciones interpersonales en los docentes educación básica. (Tesis de Maestría). Universidad Rafael Urdaneta, Maracaibo.

Stoner, J., Freeman, E. \& Gilbert, D. (1996). Administración. México: Printed.

Turiño, C., Cañizares, O. \& Sarasa, N. (2013). Necesidades de aprendizaje gerenciales en jefes de departamentos docentes de la sede central. Edumecentro, 69-76.

Mintzberg, H. (2009). Managing. Bogotá: Norma S. A. 